\title{
Size Dependent Ultrasound Characteristics in Histologically Confirmed Papillary Thyroid Carcinomas: A Multivariate Analysis
}

\author{
Michael Cordes ${ }^{1,2,4,{ }^{*}}$, Pawel Kondrat ${ }^{4}$, Karen Horstrup ${ }^{2}$, Torsten Kuwert ${ }^{1,3}$ and Marek J. Sasiadek ${ }^{4}$ \\ ${ }^{1}$ Projektgruppe Schilddrüsentumore, Tumorzentrum Universität Erlangen-Nürnberg, Germany \\ ${ }^{2}$ Radiologisch-Nuklearmedizinisches Zentrum, Nürnberg, Germany \\ ${ }^{3}$ Klinik für Nuklearmedizin, Universität Erlangen-Nürnberg, Germany \\ ${ }^{4}$ Department of Radiology, Medical University, Wroclaw, Poland
}

\begin{abstract}
Background: Epidemiologic data revealed an increasing incidence of papillary carcinomas (PTCs) in the German population. There is some evidence that the size of resected PTCs has decreased during the last few years.

Purpose: The aim of the present study was to test the hypothesis that the sonographic characteristics of PTCs vary with size.

Material/Methods: Consecutive PTCs were histologically confirmed in 41 patients. Ultrasound examinations of these 50 PTCs were reevaluated retrospectively and classified according to five sonographic criteria.

Results: It could be shown that the sonographic shape $(p<0.001)$, the contour $(p=0.024)$, the structure $(p=0.04)$, the echogenicity $(p<0.001)$ and calcifications $(p=0.008)$ varied with the size of the neoplasms. By factoranalysis a factor $F_{\text {PTC }}$ with an eigenvalue of 1.89 could be extracted from the data on which the sonographic structure, the ontour and the presence of calcifications had the strongest impact with factorloadings of $0.74,0.68$ and 0.61 , respectively.

Conclusion: The knowledge of the variation of the ultrasound characteristics should be of assistance for the sonographic classification of PTCs. In this context microcarcinomas usually do not display cystic components. However, they may show microcalcifications. Cystic components, hyperechogenicity and the taller-than-wide sign are predominantly seen in larger PTCs.
\end{abstract}

Keywords: Papillary thyroid carcinoma, ultrasound, thyroid neoplasia, irregular border, hypoechogenicity.

\section{INTRODUCTION}

According to recent data the incidence of differentiated thyroid carcinomas in Germany is increasing [1]. Essentially, this increase is caused mainly by papillary thyroid neoplasms. The occurrence of follicular neoplasms, in contrast, has remained stable over the last decades.

For the detection of thyroid nodular disease ultrasound (US) examinations of the neck play a dominant role $[2,3]$. As potential predictors for malignancy several ultrasound features have been described, such as calcifications, tallerthan-wide-shape, irregular margins, hypoechogenicity and increased flow in Power Doppler examinations [4].

The sensitivity and specificity have been found to be relatively low for each of these findings resulting in a diagnostic accuracy of far below 100\% [5].

In a recent ultrasound consensus statement the panel has identified important unanswered questions that merit future research in thyroid nodular disease [4]. Besides growth dynamics and selection of nodules for fine needle aspiration biopsy (FNAB) the panel points toward the question whether

*Address correspondence to this author at the RadiologischNuklearmedizinisches Zentrum Martin-Richter-Strasse 4390489 Nuremberg Germany; Tel: +49 911 5860148; Fax: +49 911 5860171;

E-mail: michael.cordes@rpnl.de there is a combination of ultrasound characteristics that might be used to direct management.

There is abundant scientific work using ultrasound examinations in series of thyroid nodules that include both benign and malignant neoplasms [5]. Sonographic work has also been done in malignant thyroid nodules with inconsistent different histologic differentiation [6].

Ultrasound criteria for papillary carcinomas have been compiled in a multicenter study [7]. However, this work includes only patients from a Japanese population. In a recent preliminary report from Chile a multivariate analysis was performed to identify ultrasonographic characteristics in FNAB suspected cases of papillary thyroid carcinomas [8].

The aim of the present study was to identify ultrasound characteristics in histologically proven papillary thyroid carcinomas in a caucasian population. The study was designed and carried out in order to clarify whether ultrasound patterns vary with tumor size. This work was carried out as a cohort study.

\section{MATERIAL AND METHODS}

\section{Study Population}

The study population consisted of 41 caucasian patients (30 women, 11 men, mean age +/- SD $51+/-8.7$ y; age range $17-83$ y) with 50 papillary thyroid carcinomas. All patients 


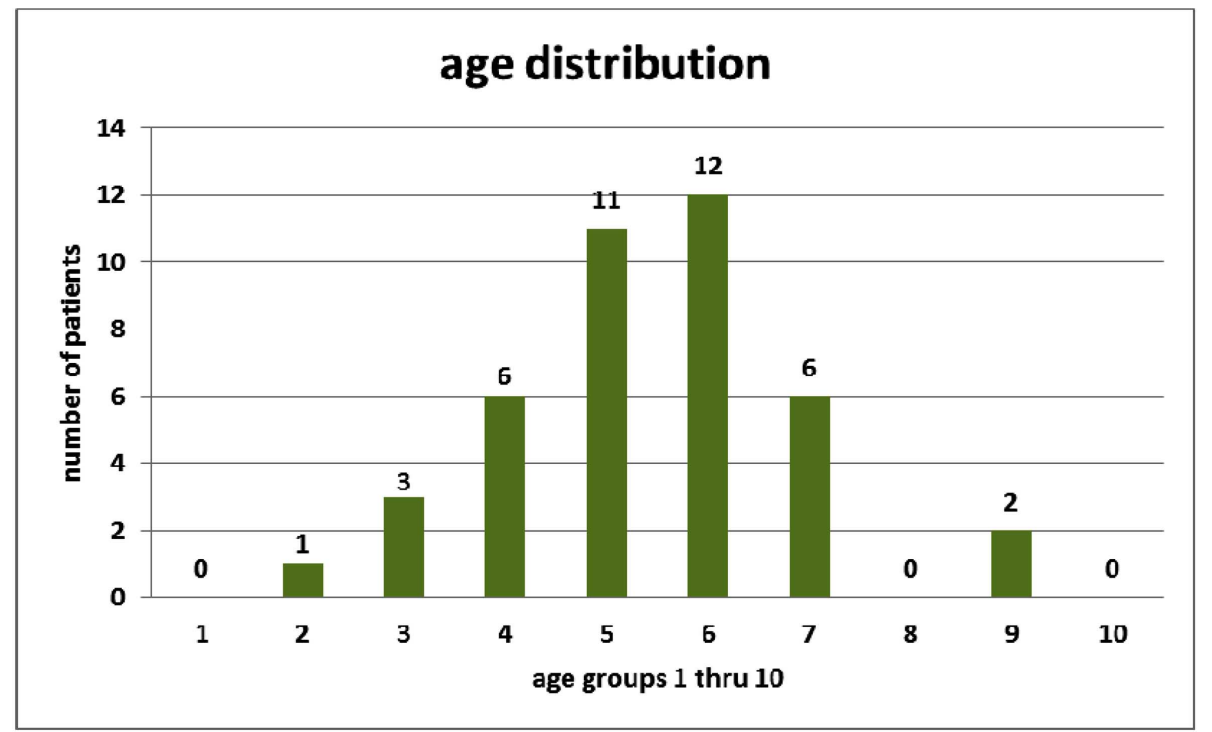

Fig. (1). Age distribution of the examined patients Age group 1: 10 - 19 years, 2: 20 - 29 years, etc.

were referred to our institution for the evaluation of thyroid nodules. The study period comprised the time between September 2010 and November 2011. Within these 15 months approx. 8000 ultrasound examinations were done.

The surgical procedures were carried out in different hospitals within 12 weeks thereafter. The diagnosis of papillary carcinoma was confirmed in all patients histologically and the histologic results were collected consecutively.

The preoperatively performed tests included a physical examination, ultrasound examinations of the cervical neck and laboratory testing in all patients. In patients with thyroid nodules $>10 \mathrm{~mm}$ in diameter a $\left[{ }^{99 \mathrm{~m}} \mathrm{Tc} \mathrm{O}_{4}\right.$ scan was performed.

\section{Ultrasound Examinations}

All ultrasound examinations of the neck were performed using an $8000 \mathrm{SE}$ Sonoace unit with a $7.5 \mathrm{MHz}$ linear transducer. US examinations were carried out by four board certified radiologists and/or nuclear medicine physicians. Representative images in two planes were digitally stored to a PACS unit. In all tumors the size was measured in three orthogonal planes by use of the implemented ultrasound cursor array resulting in a diameter in the lateral axis (dx), anterior-posterior axis (dy) and cranio-caudal axis (dz). For further evaluation and statistical analysis the maximum diameter was used.

After a total of 50 PTCs were documented by histology, image reading was reperformed retrospectively by one board certified radiologist and nuclear medicine physician. The ultrasound examinations were evaluated and described according to five sonographic characteristics.

Image reading and analysis consisted of five sonographic characteristics:

- Shape

○ round: diameter $\mathrm{dx}=\mathrm{dy}=\mathrm{dz}(+/-10 \%)$

o oval: one axis exceeds the diameter of the others by more than $10 \%$ (except taller-than-wide shape (see below)
○ irregular: undulated or complex shape

○ taller-than-wide (TTW): anterior posterior diameter > lateral diameter; cranio-caudal diameter neglected)

- Contour

$\circ \quad$ well-defined

○ spiculated or ill-defined

- $\quad$ Structure

○ homogeneous

○ inhomogeneous

- Echogenicity

o hypoechogenicity: image intensity between echofree (cystic) and below intensity of the normal perinodular tissue

○ hyperechogenicity: image intensity above normal perinodular tissue

- cystic components: echofree compartments within lesion

- Calcifications

○ present

○ absent

\section{Statistical Analysis}

Statistical analysis was performed by using the Winstat ${ }^{\mathrm{R}}$ software package (version 2009). Analysis of variance (ANOVA) and factoranalysis as multivariate tool were applied to the data. A significant difference was defined as $\mathrm{p}<0.05$.

\section{RESULTS}

\section{Age and Sex Distribution}

The patients were categorized into age groups according to their chronological age (age group 1: 10 - 19 years, age group 2: 20 - 29 years etc.) The distribution of the age groups is shown in Fig. (1). 


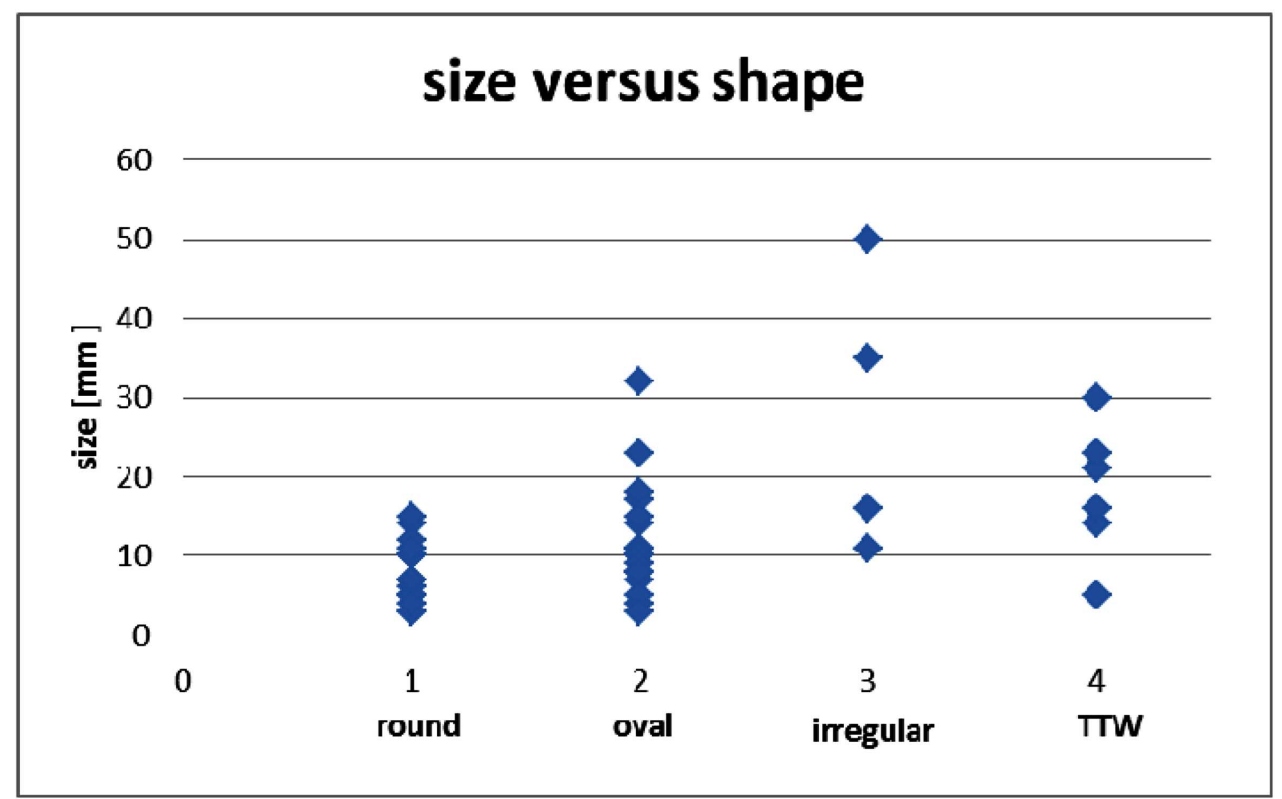

Fig. (2). Scatterplot of maximum diameter versus sonographic characteristics of PTCs.

$27 \%$ of the patients were male and $73 \%$ were female.

\section{Distribution of T Stage}

The histologic examinations of the resected specimens classified the papillary tumors as stage

$\mathrm{T} 1$ in 34 cases $(68 \%), \mathrm{T} 1 \mathrm{a}$ in 28 cases $(56 \%), \mathrm{T} 1 \mathrm{~b}$ in 6 cases $(12 \%)$

$\mathrm{T} 2$ in 3 cases $(6 \%)$

$\mathrm{T} 3$ in 10 cases $(20 \%)$ and

$\mathrm{T} 4$ in 3 cases $(6 \%)$.

Each of 32 histologic specimens showed only one PTC. Nine specimens contained two PTCs and were thus classified as multifocal (m).

\section{Ultrasonographic Characteristics}

The ultrasound examinations were evaluated with respect to the size of the PTCs and five distinct parameters. For each parameter the number of cases and their percentage were calculated.

The size of the papillary carcinomas (mean maximum diameter) varied between $3 \mathrm{~mm}$ and $50 \mathrm{~mm}$ (mean: $12.3 \mathrm{~mm}$, SD $9.0 \mathrm{~mm})$.

The echogenicity was determined as

Hypoechogenic in 40 cases $(80 \%)$

Hyperchogenic in 6 cases $(12 \%)$ and

Echofree components in 4 cases (8\%).

The shape of the tumors was

Round in 20 cases $(40 \%)$

Oval in 20 cases $(40 \%)$

Irregular in 4 cases $(8 \%)$ and

Taller-than-wide (TTW) in 6 cases $(12 \%)$.

The contour of the tumors was determined as
Well-defined in 30 cases $(60 \%)$ and

Ill-defined or spiculated in 20 cases $(40 \%)$.

The structure of the tumors was classified as

Homogeneous in 22 cases $(44 \%)$ and

Inhomogeneous in 28 cases $(56 \%)$.

Calcifications were present in 19 cases $(38 \%)$ and absent in 31 cases $(62 \%)$.

\section{Size of PTCs Versus Focality}

The mean maximum diameter of unifocal PTCs and multifocal PTCs was statistically not different $(13 \mathrm{~mm} \pm 9.5$ $\mathrm{mm}$ vs. $10 \mathrm{~mm} \pm 7.6 \mathrm{~mm})$, resp. $(\bar{x} \pm \mathrm{SD} ; \mathrm{p}=0.29$, ANOVA).

For unifocal PTCs the range of the maximum diameter was determined $3 \mathrm{~mm}$ to $50 \mathrm{~mm}$, and for multifocal PTCs 3 $\mathrm{mm}$ to $35 \mathrm{~mm}$.

\section{Size of PTCs Versus Sonographic Appearance}

The size of the PTCs as measured by ultrasound was different in round, oval, irregular and TTW tumors ( $\mathrm{p}<$ 0.001, ANOVA), see Fig. (2). The smallest mean maximum diameter was seen in round and the largest diameter in irregular tumors. Oval tumors and TTW tumors showed intermediate diameters.

Well-defined tumors showed a smaller mean maximum diameter compared with ill-defined tumors $(\mathrm{p}=0.024$, ANOVA).

The mean maximum diameter of homogeneously structured tumors was less than in inhomogeneous structured neoplasms ( $\mathrm{p}=0.04$, ANOVA).

The size of hypoechogenic, hyperechogenic and tumors with cystic components was different ( $p<0.001$, ANOVA). Hypoechogenic tumors appeared smaller than tumors with cystic components. The mean size of hyperechogenic tumors lied between hypoechogenic and cystic neoplasms. 
Table 1. Size and Ultrasound Parameters of PTCs (Mean Maximum Diameter and Standard Deviation), $\mathbf{n}=50 . \mathrm{P}$ Values are Given for ANOVA

\begin{tabular}{|c|c|c|c|c|}
\hline \multicolumn{2}{|c|}{ Ultrasound Characteristics } & Size Mean Maximum Diameter $\bar{x}[\mathrm{~mm}]$ & Standard Deviation SD [mm] & ANOVA \\
\hline \multirow{4}{*}{ shape } & round & 8 & 3.4 & \multirow{4}{*}{$\mathrm{p}<0.001$} \\
\hline & oval & 12 & 6.6 & \\
\hline & irregular & 28 & 15.5 & \\
\hline & taller-than-wide & 18 & 7.8 & \\
\hline \multirow{2}{*}{ counter } & well-defined & 10 & 7.2 & \multirow{2}{*}{$\mathrm{p}=0.024$} \\
\hline & ill-defined & 16 & 10.2 & \\
\hline \multirow{2}{*}{ structure } & homogeneous & 9 & 7.3 & \multirow{2}{*}{$\mathrm{p}=0.04$} \\
\hline & inhomogeneous & 15 & 9.5 & \\
\hline \multirow{3}{*}{ echogenicity } & decreased & 10 & 5.8 & \multirow{3}{*}{$\mathrm{p}<0.001$} \\
\hline & increased & 20 & 14.2 & \\
\hline & cystic components & 26 & 5.6 & \\
\hline \multirow{2}{*}{ calcifications } & absent & 10 & 6.7 & \multirow{2}{*}{$\mathrm{p}=0.008$} \\
\hline & present & 17 & 10.5 & \\
\hline
\end{tabular}

Tumors without calcifications showed a lesser mean maximum diameter compared with tumors that contained calcifications ( $p=0.008$, ANOVA).

No difference in size could be detected in tumors that were unifocal compared with tumors that appeared multifocal $(\mathrm{p}=0.29)$.

The sizes (mean maximum diameter) and sonographic parameters of the PTCs are shown in Table 1.

By multivariate factoranalysis the factor $\mathrm{F} 1\left(\mathrm{~F}_{\mathrm{PTC}}\right)$ with an eigenvalue of 1.89 (significance level: 1.0) was extracted from five sonographic characteristics (shape, contour, structure, echogenicity and calcifications). For these five variables the factorloading was calculated. Factors with a factorloading less than $1.0(\mathrm{~F} 2$ - F6) were neglected for further evaluation.

The sonographic structure had the highest impact on $\mathrm{F}_{\text {PTC }}$ with a factorloading of 0.74 (maximum value: 1.0 , minimum value -1.0 ), and the shape had the lowest impact on $\mathrm{F}_{\mathrm{PTC}}$ with a factorloading of $0.40(\mathrm{p}<0.05)$.

The values for the factorloadings of each variable are shown in Table 2.

\section{DISCUSSION}

In this study papillary carcinomas of less than $2 \mathrm{~cm}$ in diameter classified as stage pT1 malignancies represented 68 $\%$ of all examined tumors. In a recent publication [9] the authors pointed out that a decreasing tumor size was noticed in operated specimens. For papillary carcinomas they detected a decrease in tumor size from an average of $28 \mathrm{~mm}$ to $18 \mathrm{~mm}$ during the last 25 years.

In our series we measured an average diameter of 12.3 $\mathrm{mm}$ with a range of 3 to $50 \mathrm{~mm}$ by ultrasound examinations in papillary carcinomas that were histologically confirmed after resection. Since papillary carcinomas of less than 10 $\mathrm{mm}$ have been disclosed at operation with a proportion of 56
Table 2. Values for Factorloading for the Tested Variables by Factoranalysis $(p<0.05)$

\begin{tabular}{|c|c|}
\hline Parameter & Factorloading \\
\hline \hline structure & 0.74 \\
\hline contour & 0.68 \\
\hline calcifications & 0.61 \\
\hline echogenicity & 0.57 \\
\hline shape & 0.38 \\
\hline
\end{tabular}

$\%$ of all resected tumors, efforts should be made to characterize these microcarcinomas by appropriate ultrasound characteristics.

Unfortunately, recommendations for the management of thyroid nodules from a consensus panel excluded thyroid tumors of less than $10 \mathrm{~mm}$ [4]. Furthermore, these recommendations are extended towards all malignant thyroid nodules rather to papillary carcinomas which are the most frequent type of thyroid malignancies. This applies also to other recommendations [10] which do not specify on the different subtypes of differentiated thyroid carcinomas. However, in this work smaller tumors with a size of less than $10 \mathrm{~mm}$ have been incorporated.

Data from German and Bavarian Cancer Registries show that women are more affected than men [1, 11]. Thyroid malignancies have been reported for Germany in 2006 in $3660(69 \%)$ women and in $1620(31 \%)$ men and for Bavaria in 2008 in $972(72 \%)$ women and in 371 (28\%) men. Approximately $50 \%$ have been estimated to be papillary carcinomas. In our study, the proportion of women to men was $73 \%$ to $27 \%$ which is in agreement with the published data of the registries. The number of 50 PTCs within the collection period of 15 months, however, is relatively small. Regional features and the limited number of cases might be relevant constraints in this study. 
In 9 of 32 of our patients the resected thyroid specimens contained 2 PTCs and were thus classified as multifocal. Statistically, there was no correlation between the size of the PTCs and focality. This finding is in agreement with recently published data [12]. These authors found multicentric PTCs also in $22 \%$ of their patients. It was pointed out that in this study of 67 patients that no correlation between tumor size and multifocality existed.

Ultrasound criteria for the classification of thyroid nodules to be likely malignant have been published. These criteria include for instance a) irregular shape, b) ill-defined contour, c) solid structure and low echogenicity, d) presence of calcifications [13]. Applying citeria a) to our population would have been missed $20 \%$ of the malignant nodules, criteria b) $60 \%$, criteria c) $20 \%$ and criteria d) $60 \%$, respectively.

An irregular contour and a low echogenicity have been described as two ultrasound characteristics that yielded a 93\% sensitivity and a $92 \%$ specificity in distinguishing papillary thyroid carcinomas from benign thyroid nodules [7]. However, in this study data about the size of the PTCs that might directly influence the diagnostic accuracy are not shown. The authors used similar ultrasound characteristics compared with our study. Data about size dependent ultrasound characteristics were in this study not rendered either.

It has been proposed to combine at least two criteria in order to correctly classify sonographically detected thyroid nodules [14]. The combination of criteria a) and b) would have missed $24 \%$ of the papillary carcinomas in our study. However, if criteria c) as a third criterion is added, all carcinomas can be detected.

It is of some interest that the detection of malignant thyroid nodules by ultrasound criteria varies with size [5]. In this publication microcalcifications were found in approximately $37 \%$ of thyroid tumors less than $10 \mathrm{~mm}$ and in $51 \%$ of larger than $10 \mathrm{~mm}$. Unfortunately, this study included a heterogenious sample of papillary, follicular and medullary subtypes as well as B-cell lymphoma of the thyroid. In our study $38 \%$ and $21 \%$ of all papillary tumors or papillary tumors $</=10 \mathrm{~mm}$ contained microcalcifications, respectively.

Correlating the sonographic criteria with size shows some remarkable statistical differences. For instance, papillary tumors with irregular margins have been found to be larger than tumors with well-defined borders. This also applies to irregular shape, hyperechogenic tumors and microcalcifications in our study.

The TTW sign has been described as a manifestation of malignancy in thyroid cancer [10]. The TTW sign refers to an anterior-posterior versus transverse ratio larger than 1.0. In ultrasound examinations the TTW sign could be detected in approximately $33 \%$ of malignant thyroid tumors, including PTCs and secondary tumors of the thyroid such as metastasis of breast carcinoma [10]. It has been shown that the TTW sign of malignant thyroid nodules is more pronounced in CT examinations compared with ultrasound studies in the same patients [15]. This phenomenon might be due to compression effects of the ultrasound probe to the thyroid during the examination.
In our study the TTW sign was observed in $12 \%$ of the PTCs. The average diameter of nodules that showed the TTW sign was larger than those with round or oval shape and less than those with irregular appearance.

As a criterion for malignancy the TTW sign must be taken with caution since it has also be observed in about $8 \%$ of benign thyroid nodules [10].

It has been noted that malignant thyroid nodules exhibit several characteristics which can be detected by high resolution ultrasound examinations [16]. Recently, microcalcifications, hypoechogenicity and irregular borders have been disclosed by multivariate analysis as the variables independently associated with the presence of PTCs [8]. However, the diagnosis of PTCs was made by means of fine needle aspiration biopsy only.

From our data we extracted the factor $F_{\text {PTC }}$ by multivariate factoranalysis of five variables (shape, contour, structure, echogenicity and calcifications). The variables structure, contour and calcifications had the highest impact on the sonographic characterization of the PTCs.

In our series cystic components within the PTCs could also be observed. However, tumors with cystic components were significantly larger than other solid tumors and were not detected in the group of microcarcinomas.

In conclusion, unifocal or multifocal PTCs showed no difference in size. Even small PTCs may manifest as multifocal PTCs. Small PTCs less than $10 \mathrm{~mm}$ of diameter are frequently detected as round, hypoechogenic tumors which can contain calcifications. Hyperechogenicity or cystic components can be detected mainly in PTCs with a diameter larger than $10 \mathrm{~mm}$. PTCs characterized as TTW tumors are larger neoplasms with a diameter of more than 10 $\mathrm{mm}$ as well.

\section{CORRESPONDING AUTHORS}

Visiting Professor (Wroclaw MU) Dr. Michael Cordes.

\section{ACKNOWLEDGEMENT}

The authors thank Ms. Petra Rautenberg for the excellent preparation of the manuscript.

\section{CONFLICT OF INTEREST}

The authors confirm that this article content has no conflicts of interest.

\section{REFERENCES}

[1] Robert Koch Institut (ed.). Krebs in Deutschland 2005/2006 Häufigkeiten und Trends. Berlin. Westkreuz Druckerei 2010.

[2] Hegedüs L. Thyroid ultrasound.Endocrinol Metab Clin North Am 2001; 30: 339-60.

[3] Solbiati L, Volterrani L, Rizzatto G, et al. The thyroid gland with low uptake lesions: evaluation by ultrasound. Radiology 1985; 155: 187-91.

[4] Frates MC, Benson CB, Charboneau JW, et al. Management of thyroid nodules detected at US: Society of Radiologists in US consensus conference statement. Radiology 2005; 237: 794-800.

[5] Moon WJ, Jung SL, Lee JH, et al. Benign and malignant thyroid nodules: US differentiation- multicenter retrospective study. Radiology 2008; 247: 762-70.

[6] Tae HJ, Lim DJ, Baek KH, et al. Diagnostic value of ultrasonography to distinguish between benign and malignant 
lesions in the management of thyroid nodules. Thyroid 2007; 17: 461-6.

[7] Shimura H, Haraguchi K, Hiejima Y, et al. Distinct diagnostic criteria for ultrasonographic examination of papillary thyroid carcinoma: a multicenter study. Thyroid 2005; $15: 251-8$.

[8] Dominguez JM, Baudrand R, Arteaga E, et al. An ultrasound score to predict the presence of papillary thyroid carcinoma. Preliminary report. (Paper in Spanish). Rev Med Chile 2009; 137: 1031-6.

[9] Machens A, Dralle H. Decreasing tumor size of thyroid cancer in Germany: institutional experience 1995 - 2009. Eur J Endocrinol 2010; 163: 111-9

[10] Kim EK, Park CS, Chung WY, et al. New sonographic criteria for recommending fine-needle aspiration biopsy of nonpalpable solid nodules of the thyroid. Am J Roentgenol 2002; 178: 687-91.

[11] Bevölkerungsbezogenes Krebsregister Bayern 2011, Registerstelle. Available at: www.krebsregister-bayern.de [Access March 20, 2012].
[12]

Schönberger J, Marienhagen J, Agha A, et al. Papillary microcarcinoma and papillary cancer of the thyroid $<=1 \mathrm{~cm}$. Nuklearmedizin 2007; 46: 115-20.

[13] Paschke R, Hegedüs L, Alexander E, Valcavi R, Papini E, Gharib $\mathrm{H}$. Thyroid nodule guidelines: agreement, disagreement and need for future research. Nat Rev Endocrinol 2011; 7(6): 354-61.

[14] Paschke R. Abklärung des euthyreoten Schilddrüsenknotens: wann punktieren? Stellenwert der Sonographie. Dtsch Med Wochenschr 2009; 134: 2498-503.

[15] Yoon SJ, Yoon DY, Chang SK, Seo YL, Yun EJ, Choi CS Bae SH. "Taller-than-wide" sign of thyroid malignancy: comparison of ultrasound and CT. Am J Roentgenol 2010; 194: W420-4.

[16] Hoang JK, Lee WK, Lee M, Johnson D, Farrell S. US features of thyroid malignancy: pearls and pitfalls. Radiographics 2007; 27: $847-65$.

(C) Cordes et al.; Licensee Bentham Open.

This is an open access article licensed under the terms of the Creative Commons Attribution Non-Commercial License (http://creativecommons.org/licenses/bync/3.0/), which permits unrestricted, non-commercial use, distribution and reproduction in any medium, provided the work is properly cited. 